\title{
UV laser-induced high resolution cleaving of Si wafers for micro-nano devices and polymeric waveguide characterization
}

\author{
R. Casquel ${ }^{\mathrm{a}, *}$, M. Holgadoa , J.J. García-Ballesteros ${ }^{\mathrm{a}}$, K. Zinoviev ${ }^{\mathrm{b}}$, C. Férnandez-Sánchez ${ }^{\mathrm{b}}$, \\ F.J. Sanza ${ }^{a}$, C. Molpeceres ${ }^{a}$, M.F. Laguna ${ }^{a}$, A. Llobera ${ }^{\text {b }}$,.L. Ocaña ${ }^{a}$, C. Domínguez ${ }^{b}$ \\ ${ }^{a}$ Centro Láser UPM, Universidad Politécnica de Madrid, Campus Sur UPM, 28031 Madrid, Spain \\ b Instituto de Microelectrónica de Barcelona, Centro Nacional de Microelectrónica - CSIC, Campus Universidad Autónoma de Barcelona, 08193 Bellaterra, Barcelona, Spain
}

Keywords:

Laser cleaving

Micro-nano photonic chips

Polymeric waveguides

Silicon fracture

\begin{abstract}
A B S T R A C T
In this work we propose a method for cleaving silicon-based photonic chips by using a laser based micromachining system, consisting of a ND: $\mathrm{YVO}_{4}$ laser emitting at $355 \mathrm{~nm}$ in nanosecond pulse regime and a micropositioning system. The laser makes grooved marks placed at the desired locations and directions where cleaves have to be initiated, and after several processing steps, a crack appears and propagate along the crystallographic planes of the silicon wafer. This allows cleavage of the chips automatically and with high positioning accuracy, and provides polished vertical facets with better quality than the obtained with other cleaving process, which eases the optical characterization of photonic devices.

This method has been found to be particularly useful when cleaving small-sized chips, where manual cleaving is hard to perform; and also for polymeric waveguides, whose facets get damaged or even destroyed with polishing or manual cleaving processing. Influence of length of the grooved line and speed of processing is studied for a variety of silicon chips. An application for cleaving and characterizing sol-gel waveguides is presented. The total amount of light coupled is higher than when using any other procedure.
\end{abstract}

\section{Introduction}

Silicon based mass production technology at the micro/ nanoscale is increasing the number of devices every year. This miniaturization and integration is broadening the functionality of micro-nano devices rapidly for a wide field of applications such as micro-nano systems, MEMS, MOEMS, lab-on-a-chip, or sensing devices [1-3]. In this term, high resolution wafer dicing and cleaving is a significant feature in order to control both the fabrication processes and the functionality characterization of the devices. Initial fabrication steps are usually implemented on a Si wafer that contains a large number of devices. After this, quite often the wafers have to be cleaved to analyze the vertical facets to control the fabrication processes or, in the case of photonic devices, to make accessible input and output waveguides in order to evaluate their optical response. However, although wafer dicing and polishing is a reliable process for this purpose, it is time consuming and a significant know-how is needed to implement at micro-nano scale.

In this work we propose an alternative procedure for cleaving, using a Nd: $\mathrm{YVO}_{4}$ laser emitting pulsed UV radiation at $355 \mathrm{~nm}$ in nanosecond pulse regime and a high resolution positioning system for cleaving without human manual intervention. The laser is used for making grooved marks that help on initiating the cleavage crack formation. The formation of the grooves provides a well-controlled mechanism for a clean crack formation through the material to reach sub-micro domains in micro-nano structures fabricated over Si wafer for their analysis. In particular, we demonstrate the benefit of this procedure for polymeric waveguides [4], in which the polishing may produce strong damage, reducing the optical output until making it negligible in some cases. Results of measurements are higher for laser cleaving process than for any alternative process employed.

Cleavage of silicon devices is a task often performed manually, using diamond scribers, or dicing the wafer with a tool, and finally polishing the surface. By using a laser beam any physical contact with the device is avoided, which can be a significant improvement. Studies in the dynamics of crack formation, when using laser-based techniques, have been taken out, both from experimental and theoretical point of view [5-9]. In a general way, the cleaving is applied to brittle materials, such as glass, ceramic materials, and silicon wafers. For example, Lin and collaborators used a line-shaped beam [7] and a combination of line-shaped beam and a circular beam pulsed laser [8] to cleave a sheet of glass, whereas Ueda et al. [5] used a laser beam scanning at a certain speed to generate cleaves on a silicon wafer and ceramic materials 


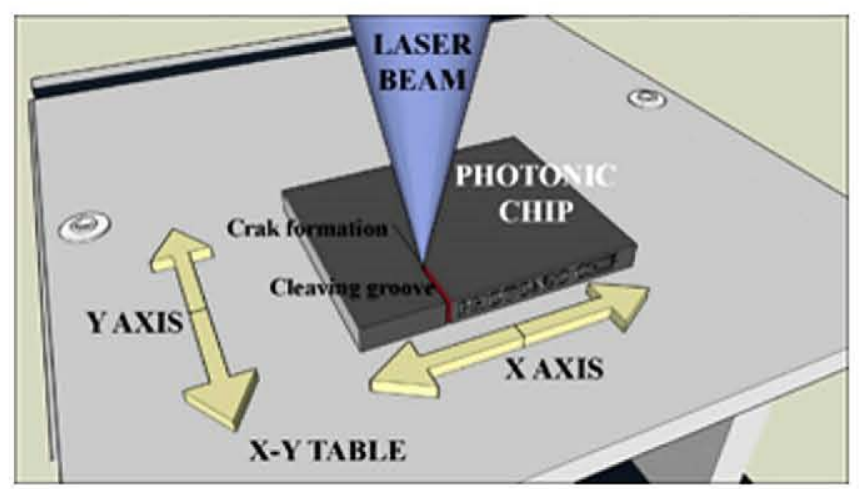

Fig. 1. Schematic of the experimental setup.

such as alumina and zirconium. They used a circular spot size of $650 \mu \mathrm{m}$ (Ueda) and a line shape beam of $15 \mathrm{~mm} \times 0.7 \mathrm{~mm}$ (Wang). Finally, Tsai and Liou [9] studied the fracture dynamics for several materials, thicknesses, spot sizes and the reached stability of the crack formed, and the reasons of non-stable cracks.

In contrast with these works, we used a laser with a micrometric spot size, in the order of $20 \mu \mathrm{m}$, empowered with a micropositioning system. The reason for using a micrometric spot is to achieve a highly precise positioning of cleavage planes. This is of high importance when characterizing photonic chips, as their size is often in this range: for example we find resonant rings with a diameter in the order of $100 \mu \mathrm{m}$ in diameter [3], or Distributed Bragg Reflectors (DBR) with a cavity length of $20 \mu \mathrm{m}$. With a spot size of this order, and a precision in positioning of $1 \mu \mathrm{m}$, we are able to ensure the cleavage of a device with a size of $50 \mu \mathrm{m}$ or even smaller.

The particularity of silicon, compared to other brittle materials, is that it is crystalline, with a diamond-like lattice; silicon wafers are generally classified depending on the orientation of the crystal; most common wafers are (111), (100) or (110). Cracks are generated mainly along these planes, as the required energy to break up is significantly lower compared with any other direction [10]. The dynamics of the generation of the crack have been detailed in the literature [11].

\section{Experimental process}

The cleavages were carried out with a laser-based micromachining system (Optec ML-100), consisting of two laser sources emitting UV light within nanosecond pulse duration regime (solid state and excimer lasers). A $X-Y$ positioning table with a spatial resolution of $1 \mu \mathrm{m}$ is also included, besides of a CCD camera. The laser used for these experiment is an optically pumped (with laser diodes) ND:YVO4 laser, with these characteristics: $\mathrm{aTEM}_{00}$ Gaussian beam, maximum power of $5 \mathrm{~W}$, repetition rate from $5 \mathrm{kHz}$ to $150 \mathrm{kHz}$, pulse duration of $12 \mathrm{~ns}$, emitting at $355 \mathrm{~nm}$. Light is focused on the sample with a spot size of $20 \mu \mathrm{m}$ through a $0.15 \mathrm{NA}$ focusing lens. The system has been used for several applications, mainly related with microprocessing materials such as amorphous silicon and ITO. In [12] are detailed these process besides the main characteristics of the system.

Fig. 1 shows a schematic representation of the experimental procedure used. The laser focuses on the sample, and scans along the $Y$ direction with a speed $v$. A linear groove is created in the surface of the chip, with a length $L$. After several scannings, the crack appears, and it propagates along the grooved line direction as the scan continues. The properties of the beam depend strongly on the optical pumping and the repetition rate. For our experiments we chose $15 \mathrm{kHz}$ and current of the diode pumping at $75 \%$ of the maximum current as these parameters provide a higher beam quality, although lower average energy ( $2 \mathrm{~W}$ ) within a smaller spot diameter ( $20 \mu \mathrm{m}$ compared with $40 \mu \mathrm{m}$ at maximum power).

Previous works mentioned in Section 1, as the cleaving of silicon by Ueda et al. [5] and the line-shaped beams from Lin [7,8] showed that the laser heating induces compressive stresses around the laser spot, which are relaxed when the laser stops, and then turn into induced residual stresses, which produce the crack when a threshold value is reached. So a moving laser source is used, and the cracking starts in an area after the scanning of the laser. Also, calculations based on finite element software $[7,8]$ showed that the stress state produced on the focused element is complex, and shearing stresses play an important role in the crack formation.

In our case, we could not generate clean cracks just by heating the surface of the chips, due to different parameters (smaller spot sizes, smaller energy, different wavelength, lower speed of scanning available, among others). So our strategy consisted of generating grooves along the crystalline planes, scanning several times with the laser at a certain speed $(v)$. These grooves define exactly the planes where we want the crack to appear. In the first scannings, the incident energy is used in removing material. After this, the sample is just heated. However, as the geometry has changed, also change the stress distribution, and the end of the groove is an area where stress can have a multiplying factor, similar to the classic problem of a notch in a sample, for example explained in the work of Pilkey [13]. This suggests that the crack is more likely to start at the end of this groove, as schematically drawn in Fig. 1, and this is what happened into the majority of our experiments. Hauch et al. described a similar phenomenon in their work [11], where the speed of formation of the crack is higher as the crack length increases.

The steps for the process are the following: the first stage consists of positioning the sample into the micromachining tool. We have to ensure that the groove created is parallel to the crystallographic plane of the silicon chip. If this is done properly, the shearing stresses dominating the crack formation will be also contained in a crystallographic plane, making absolute value of stress needed for cracking lower. These planes came defined by the flat of the wafer. For this purpose we have a goniometer, so the sample is turned a certain angle until the wafer flat is parallel to one of the scanning axes. After this, we make the laser grooves on the material, with a scanning speed $v$ and a length of the groove $L$. As the energy density is quite high, in the first laser scannings the energy incident to the wafer removes material and creates the groove. Finally, after several scannings, the crack appears, and propagates along a crystallographic plane. When the groove is oriented parallel to one of this planes, the crack propagates faster, otherwise the crack and the groove will form a small angle, with slower propagation. At a certain number of laser processes, the crack stops and there is no more advance. However, the cleavage plane is well defined, and just by moving the chip, it gets divided into two different parts. Fig. 2 shows a cleaved chip, and the comparison between grooved area and where the crack appears. The cleaved surface reached is highly smooth, with high stability of formation of the crack (there is no significant change in the direction of growth).

\section{Results}

We have cleaved a wide variety of Si-based chips using this procedure. Among all the parameters to be optimized within the process, we have found the most important ones to be the length of the groove and the speed of processing. We cannot determine solely the influence of repetition rate in the process, as a change in this rate provokes a change in beam properties also (quality, shape and size of the beam). For this reason, $15 \mathrm{kHz}$ and maximum power at this rate $(2 \mathrm{~W})$ are constant for all the experiments, as this 


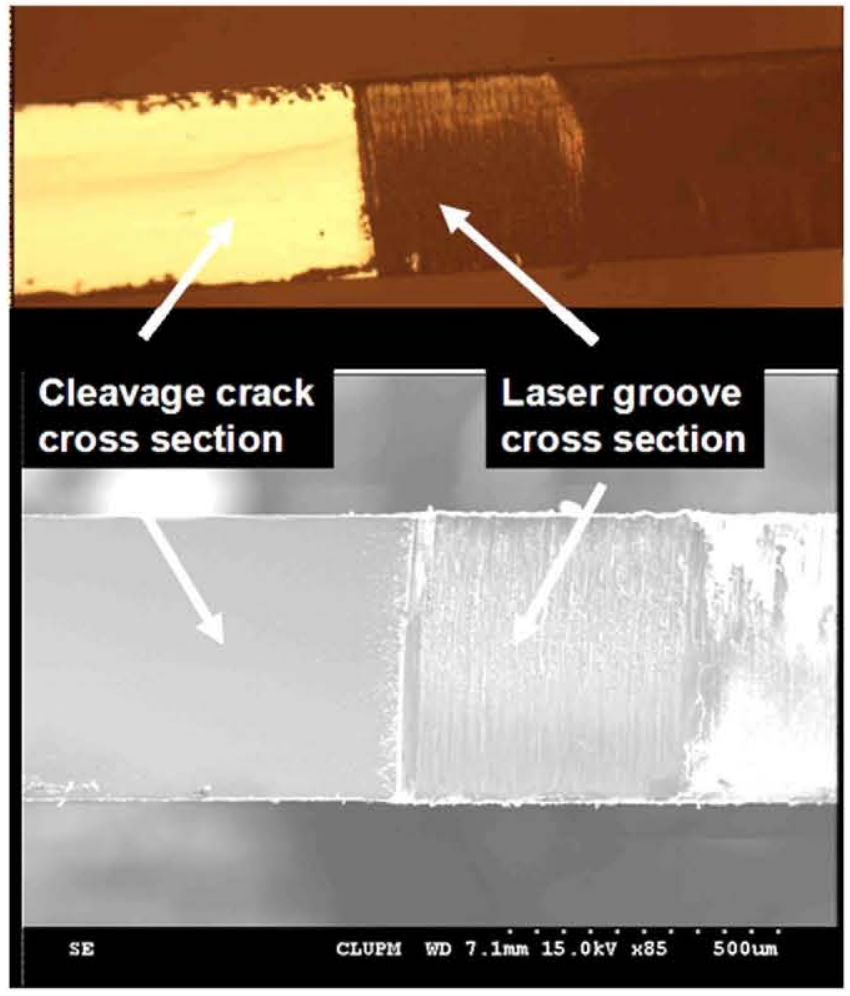

Fig. 2. Optical microscope and SEM cross sections of the laser grooved and cleavage areas.

configuration provides the better beam quality, furthermore. The scanning speed is between 15 and $100 \mu \mathrm{m} / \mathrm{s}$. The processing speed can have influence in the speed of generation of the crack, as Ueda et al. [5] reported. However, they used a speed between $1 \mathrm{~mm} / \mathrm{s}$ and $36.7 \mathrm{~mm} / \mathrm{s}$, compared with the speed of $100 \mu \mathrm{m} / \mathrm{s}$ used in this work, which is several orders of magnitude higher.

Fig. 3 shows the laser procedure for two different processes. Fig. 3a represents a processing speed of $15 \mu / \mathrm{s}$, whereas in Fig. 4b this speed is $100 \mu / \mathrm{s}$. The groove length is for both cases of $2 \mathrm{~mm}$. In (a) crack appears in the scanning number 4 (533 s of total scanning), and in (b) in number 10 (200s), and the total length of the crack is higher in the second case, besides of having a more linear growth. This suggests a better behavior of the cleavage when scanning speeds are higher.

Also, a higher length of the groove also eases the crack showing up. This is in accordance with stress concentration factor calculation [13]. Not only the total length of the groove is important, but also the relation between length groove and total length of the chip, as noted in the equation of stress concentration. How- a

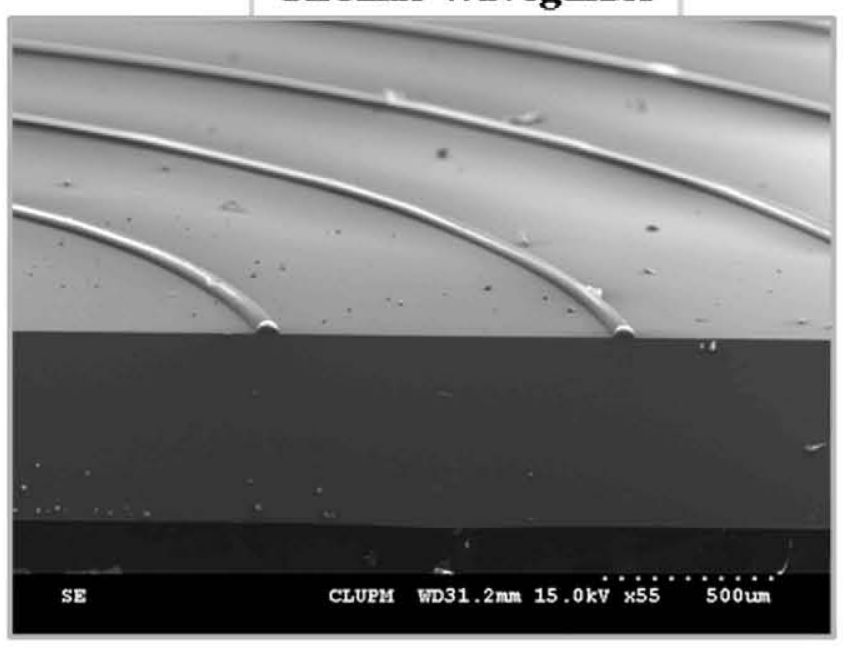

b

\section{Linear waveguides}

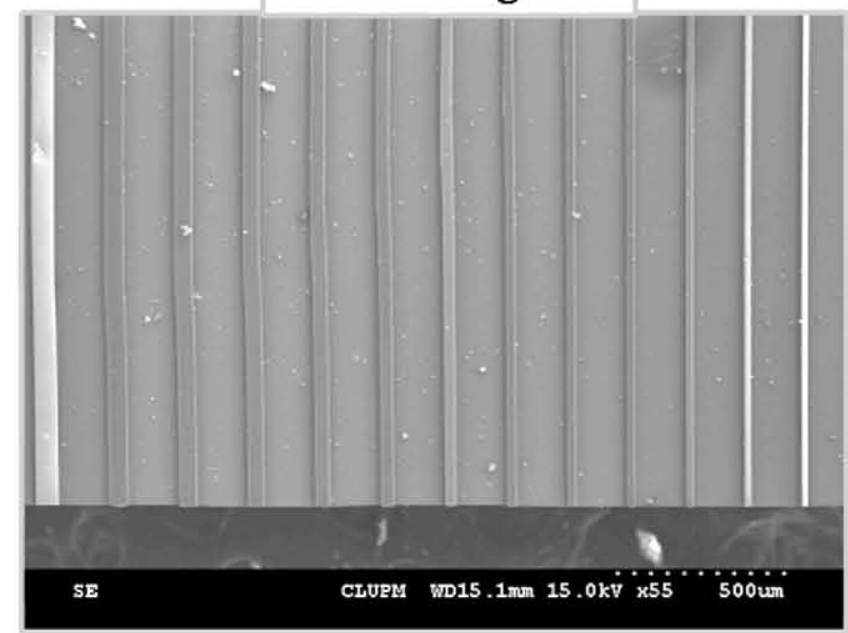

Fig. 4. Cleaved chip with a set of sol-gel waveguides with different thickness on silicon. Captions using (a) optical microscopy and (b) scanning electron microscopy.

ever, the length of the groove has practical limitations. The chips to be cleaved have often reduced size, sometimes smaller than $10 \mathrm{~mm} \times 10 \mathrm{~mm}$. The groove must be made in an area where there is no photonic structure, as the laser scanning would damage them. So grooves are limited to a length generally in the order of $2 \mathrm{~mm}$
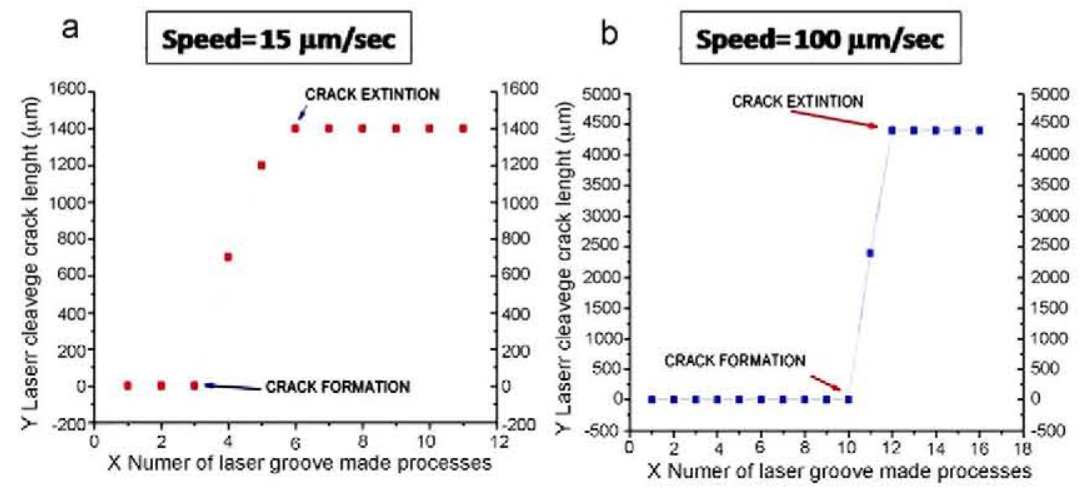

Fig. 3. Number of laser groove-made processes vs. cleavage crack length. 
or lower. However, this length has demonstrated to be enough for most of the cleaved chips.

It must be noted that the cleaved chips, or the chips intended to be cleaved in the future, can be built over different type of silicon wafers (crystal orientation, doping, thickness), and with layers of several materials (dielectrics, metals, polysilicon), variations that have influence in the fracture limit of the chip [14]. Besides this, the size of the chips is not uniform, and neither is the available space for making the groove on it. So we have tried to develop a standard process to cleave a wide variety of chips. A process consisting of 10 scans at a speed of $100 \mu \mathrm{m} / \mathrm{s}$, with a length over $2 \mathrm{~mm}$ has proved to work in almost all varieties of chip.

In particular, Fig. 4 shows an example of two chips with a set of sol-gel waveguides on them, the first one (a) with circular waveguides, and the other with linear ones (b). These are polymeric waveguides, in which laser cleavage is under our point of view of high interest, as the quality of the facets has critical importance to have any signal during the photonic characterization.

The chip in Fig. 4b consists of several sol-gel waveguides, with a height of $35 \mu \mathrm{m}$, a length close to $10 \mathrm{~mm}$, and width up to $200 \mu \mathrm{m}$. These sol-gel polymer waveguides were fabricated as described elsewhere with slight modifications [15]. They should be cleaved in order to release their facets since an excess of the sol-gel material appeared at both ends of the structures due to the MIMIC fabrica-

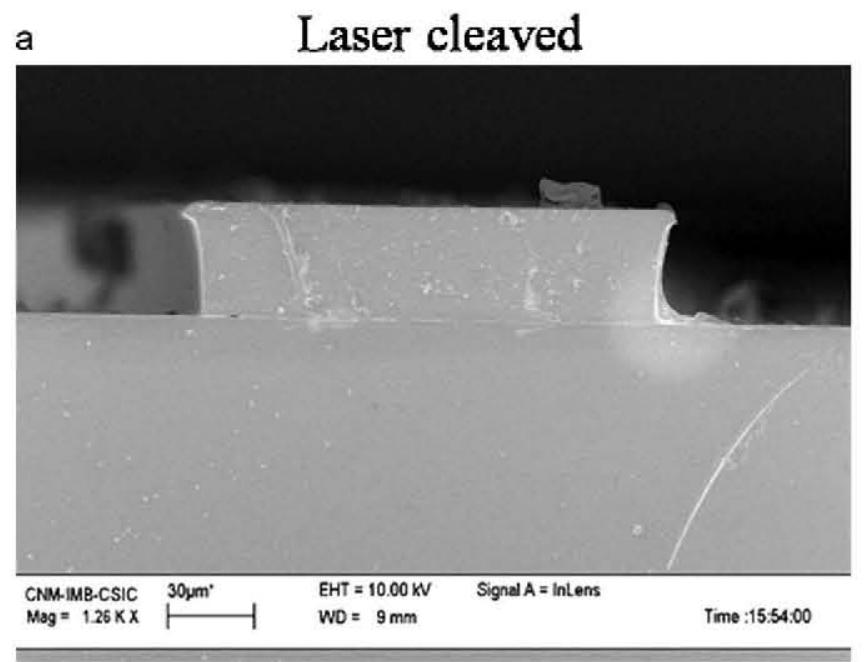

b

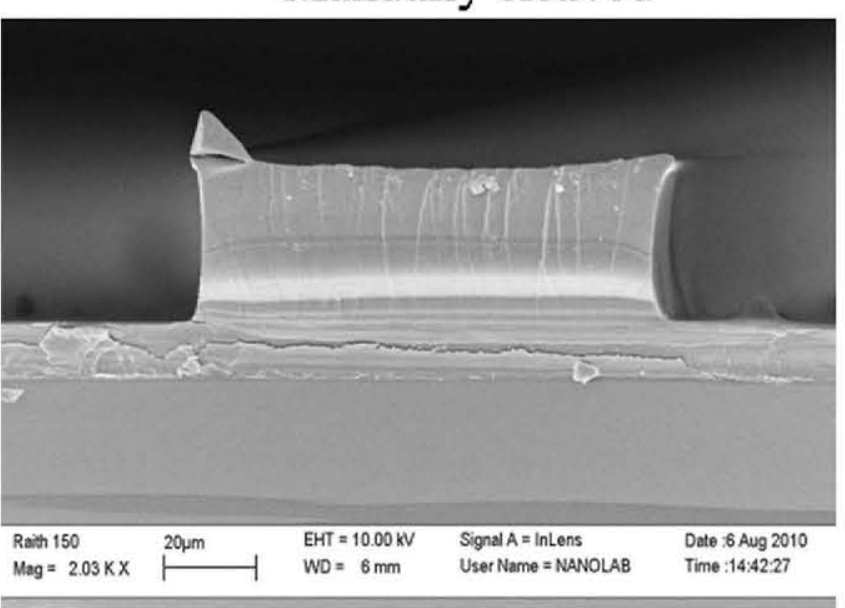

Fig. 5. SEM images of the facets of sol-gel polymer waveguides. (a) Laser cleaved. (b) Manually cleaved. Quality of facets of laser cleaving is much higher.

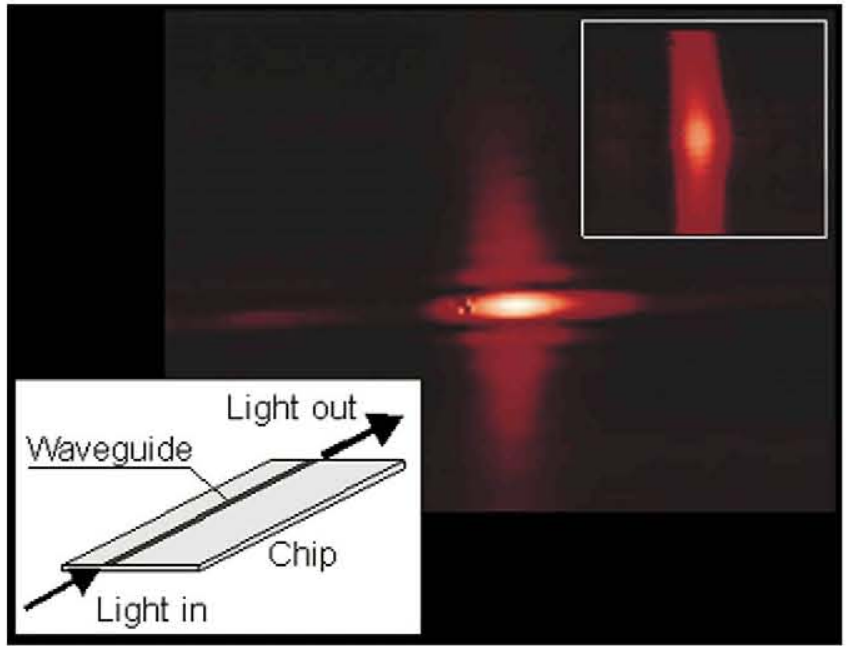

Fig. 6. Qualitative estimation of the waveguide facets formed by laser cleaving. The light exiting the waveguide has a smooth intensity distribution what is an evidence of good quality of the facet.

tion process [16]. Fig. 5 shows SEM images of the facets of sol-gel waveguides manually and laser cleaved. Clearly, with laser cleavage the facets are of higher quality. In (b) seems that the crack has propagated along different crystalline planes, in contrast with (a). Manual cleavage would be good enough as long as these were not photonic waveguides.

The waveguides became fully accessible after cleaving and we managed to couple about $20 \%$ of HeNe laser light $(632.8 \mathrm{~nm})$ focused by an objective lens on the waveguide facet (19.3 for $100 \mu \mathrm{m}$ waveguide, and 21.3 for $150 \mu \mathrm{m}$ ). This has been higher in any case to the efficiency usually obtained when we couple light using the same method into the waveguides with manual cleaving ( 7.15 for $100 \mu \mathrm{m}$ and 4.25 for $150 \mu \mathrm{m}$ ). Besides, yield for laser cleaved waveguides was of $90 \%$, compared to $66 \%$ with manual cleaving.

The quality of the chip facet might be estimated by intensity distribution of light exiting the cleaved waveguide. Light exiting the waveguide was projected by an objective lens with NA 0.65 onto a CCD camera. An image of the exiting mode of light with TE polarization is presented in Fig. 6 . The mode is continuously distributed, which is an evidence of high quality of waveguide facet.

\section{Conclusions}

We have developed a methodology to cleave Si-based chips using a laser micromachining tool. Accuracy and resolution in generating the cracks and high quality of facets of the photonic devices are main advantages of this method. Highly smooth surfaces are reached, with the advantage of reducing risk of damaging photonic waveguides, as the laser scanning is performed at a distance of them. A particular application for a polymeric sol-gel waveguide is also presented. The amount of light coupled to the waveguide is higher (up to four times) than those obtained using other methods (manual cleavage, dicing and polishing).

\section{Acknowledgements}

Funding was provided by the EU Project PHODYE (STREPFP6-IST-0033793), BIOPSIA project under the Spanish Ministry of Science and Innovation (REF: TEC2008-06574) and the FPU program of the Technical University of Madrid. 


\section{References}

[1] P.N. Prasad, Nanophotonics, Wiley, 2004.

[2] C.A. Barrios, M.]. Bañuls, V. González-Pedro, K.B. Gylfason, B. Sánchez, A. Griol, A. Maquieira, H. Sohlström, M. Holgado, R. Casquel, Label-free optical biosensing with slot-waveguides, Opt. Lett. 33 (2008) 708-710.

[3] M.E. Motamedi, Micro-opto-electro-mechanical systems, Opt. Eng. 33 (1994) $3505-3517$.

[4] H. Ma, A. Jen, L.R. Dalton, Polymer-based optical waveguides. Materials, processing and devices, Adv. Mater. 14(2002) 1339-1365.

[5] T. Ueda, K. Yamada, K. Oiso, A. Hosokawa, Thermal stress cleaving of brittle materials by laser beam, Ann. CRIP 51 (2002) 159-162.

[6] A. Saimoto, Y. Imai, F. Motomura, Simulation of crack growth in thermal stress cleaving using line heat source, JSME J. Ser. A 42 (1999) 578-584.

[7] Y. Wang, J. Lin, Characterization of the laser cleaving on glass sheets with a line-shape laser beam, Opt. Laser Technol. 39 (2007) 892-899.

[8] Y.L. Kuo, J. Lin, Laser cleaving on glass sheets with multiple laser beams, opt. Laser Eng. 46 (2006) $388-395$.
[9] C.H. Tsai, C.H. Liou, Fracture mechanism of laser cutting with controlled fracture, J. Manuf. Sci. Eng. 123 (2003) 519-528.

[10] K.E. Petersen, Silicon as a mechanical material, Proc. IEEE 70 (1982) $420-448$.

[11] J. Hauch, D. Holland, M.P. Marder, H.L. Swinney, Dynamic fracture in single crystal silicon, Phys. Rev. Lett. 82 (2008) 3823-3826.

[12] C. Molpeceres, S. Lauzurica, J.L. Ocaña, J.J. Gandía, L. Urbina, J. Cárabe, Microprocessing of ITO and a-Si thin films using ns laser source, J. Micromech. Microeng 15 (2005) 1271-1278.

[13] W. Pilkey, Stress Concentration Factors, John Wiley and Sons, 1997.

[14] S. Johansson, F. Ericson, J.A. Scheitz, Influence of surface coatings on elasticity, residual stresses, and fracture properties of silicon microelements, J. Appl. Phys. 65 (1989) 122-128.

[15] A. Llobera, VJ Cadarso, M. Darder, C. Domínguez C. Fernández-Sánchez, Fullfield photonic biosensors based on tunable bio-doped sol-gel glasses, Lab Chip 8 (2008) 1185-1190.

[16] E. Kim, Y. Xia, G.M. Whitesides, Micromolding in capillaries: applications in materials science, J. Am. Chem. Soc. 118 (1996) 5722-5731. 